\title{
Angular momentum in general relativity: The definition at null infinity includes the spatial definition as a special case
}

\author{
Anthony Rizzi \\ California Institute of Technology, LIGO, Livingston, Louisiana 70754 \\ (Received 7 February 2000; published 4 April 2001)
}

\begin{abstract}
I show that the definition of angular momentum at null infinity given by A. Rizzi, Phys. Rev. Lett. 81, 1150 (1998), reduces to the Arnowitt-Deser-Misner definition at spatial infinity in the appropriate limit. This identification reinforces the gauge condition of the null definition as well as the null definition itself. Insight into the place of the null and spatial definitions is also gained. The methods used in this paper are very useful for understanding far-field problems in general relativity; a set of Appendixes gives details of the methods.
\end{abstract}

DOI: $10.1103 /$ PhysRevD.63.104002

PACS number(s): 04.20.Cv, 04.20.Ha

\section{INTRODUCTION}

Until recently, there was no definition of angular momentum in general relativity that allowed for a nontrivial conservation law. The only definition of angular momentum that was consistent within general relativity was the ArnowittDeser-Misner (ADM) spatial definition [1,2,3]; this definition gave an angular momentum for the entire space-time. The ADM definition was only marginally useful, because it allowed no way of speaking of the exchange of angular momentum. That is, one could not even make simple conservation statements, such as a system with angular momentum $L$ gave up $\Delta L$ and was thereby left with $L-\Delta L$. Previous attempts at defining this basic quantity in a satisfactory way failed for various reasons; prime among these reasons was the so-called supertranslation ambiguity. These problems were solved in Ref. [4]. In the present paper, I will show that the definition of angular momentum at null infinity given in [4] reduces to the ADM definition ${ }^{1}$ in the appropriate limit. The agreement between the two definitions strengthens our overall understanding of angular momentum in general relativity. Since the ADM definition is well established it lends another successful test to the definition given in [4]. Furthermore, it gives context to the ADM definition. To avoid wordiness, henceforth the definition given in [4] will be referred to as the null-AM definition or simply the null definition.

The most intuitive way of seeing that there is a point where the definition at null infinity meets the spatial definition is to make use of the conformal picture. In the conformal picture one maps infinity to a finite location. The conformal picture of an asymptotically flat space-time is shown in Fig. 1. The null definition of angular momentum exists along the axis labeled $\mathfrak{I}^{+}$, "Scri+,", spatial infinity is shown as a small circle marked $i^{0}$.

Mathematically, one says

\footnotetext{
${ }^{1}$ By "ADM definition," I mean what is canonically taken to be the ADM definition. The ADM definition is only clear and unambiguous when given in the center of mass frame $\left(P_{C M}=0\right)[5,6,7]$. Therefore, in this paper, "ADM definition" refers to the ADM equation taken in this case.
}

$$
\lim _{u \rightarrow-\infty} L_{\text {null }}(u)=L_{\text {spatial }}
$$$$
\text { where } u \text { parametrizes } \mathfrak{I}^{+} \text {. }
$$

To make this mathematically precise, we recast the spatial and null definitions in terms of a space-time foliation adapted to the appropriate limits. We will work in strongly asymptotically flat (SAF) space-times (cf. Appendix A).

We use the optical-maximal foliation. This is my name for the foliation used in Christodoulou and Klainerman's book [8] on the nonlinear stability of the Minkowski space. As the name suggests, the optical-maximal foliation is the $S^{2}$ foliation (an $S^{2}$ foliation is one with leaves that are topologically $S^{2}$ ) that results from the intersection of an "optical" foliation and a "maximal foliation."

A maximal slice of space-time, designated by time $t, \Sigma_{t}$, is a spatial slice which has maximal volume with respect to the embedded space; mathematically this means $\operatorname{tr}\left(k_{i j}\right)=0$ where $k_{i j}$ is extrinsic curvature of $\Sigma_{t}$. One must impose two

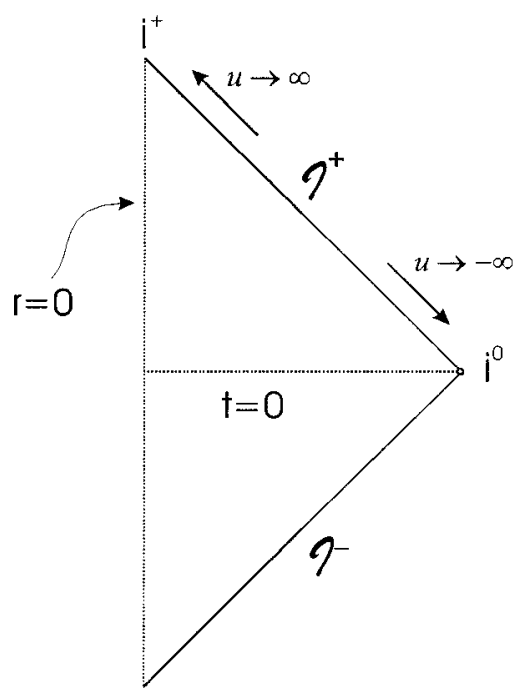

FIG. 1. The null definition of angular momentum is valid all along $\mathfrak{I}^{+}$which should include spatial infinity, $i^{0}$. Hence, if the limit is taken along $\mathfrak{I}^{+}$to $i^{0}$, one should get the spatial definition also know as the ADM definition. 


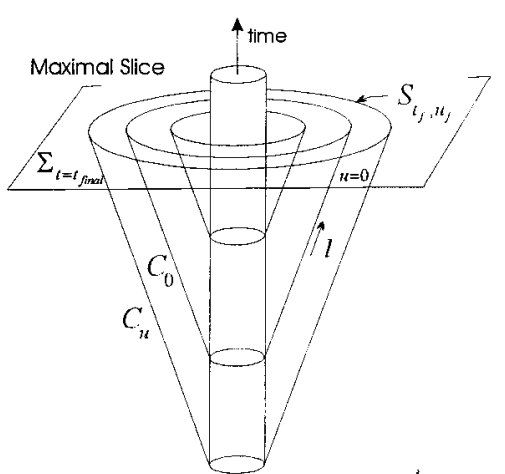

FIG. 2. The optical foliation is shown starting with one maximal slice. This "final" slice starts out at finite time, $t$, and then a limit is taken to the infinite future. The optical foliation is a null 2-surface folation.

more constraints to make the slicing of space-time unique: (1) pick an ADM linear momentum for the slice and (2) choose the metric such that the lapse function, ${ }^{2} \phi$, is one at spatial infinity (i.e., $t$ becomes proper time at spatial infinity). We need the zero ADM momentum (center of mass frameSAF conditions imply data set in $P_{\mathrm{ADM}}=0$ ). Assigning such a maximal hypersurface, $\Sigma_{t}$ for each $t$ generates what will be called the "maximal foliation."

The optical foliation (cf. Chaps. 9 and 14 of [8]) is a null 2 -surface foliation. It is defined everywhere far from the region of mass so that geodesics are, in a suitable sense, wellbehaved. That is, the foliation is defined everywhere exterior to a time-like cylinder enclosing the mass. The foliation is shown in Fig. 2. To create the foliation we do the following.

(a) Start with a maximal hypersurface, $\Sigma_{t=t_{\text {final }}}$, of zero ADM momentum and construct a 1-parameter family of $S^{2}$ surfaces (i.e. surfaces which are diffeomorphic to $S^{2}$ ) on $\Sigma_{t=t_{\text {final }}}$ by solving a given equation of motion (cf. [8], p. 411) on $\Sigma_{t=t_{\text {final }}}$. This process gives us the function $u_{f}$ defined on $\Sigma_{t=t_{\text {final }}}$ whose level surfaces will be labeled by $S_{t_{f}, u_{f}}$.

(b) For a given $S_{t_{f}, u_{f}}$ of $\Sigma_{t_{\text {final }}}$ send light rays into the past thus creating a null cone called $C_{u}$. If one does this for each $S_{t_{f}, u_{f}}$, he extends the optical function $u_{f}$ on $\Sigma_{t_{\text {final }}}$ to an optical function $u$ in the space-time.

(c) To make the solution valid for all space-time outside the timelike cylinder one lets $t_{\text {final }} \rightarrow \infty .^{3}$ This gives an optical

\footnotetext{
${ }^{2}$ One can write the space-time metric as $d s^{2}=-\phi^{2} d t^{2}$ $+g_{i j} d x^{i} d x^{j}$ where $\phi$ is called the lapse function.

${ }^{3}$ In taking this foliation to time-like infinity, one is making the physically reasonable assumption that the entire space-time exists (global existence). However, since the proof given in this paper takes place "near" spatial infinity, infinitely far away from timelike infinity, one does not, even in a technical sense, need the proof of global existence here. In a word, the foliation is extended to time-like infinity only to give a complete intuitive picture of how the space-time is sliced.
}

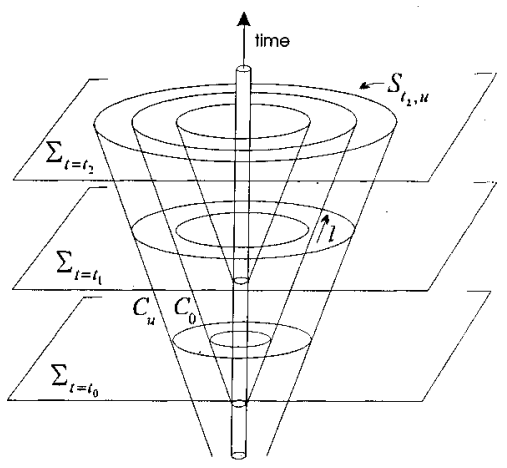

FIG. 3. The optical-maximal foliation is a foliation of spacetime with topologically $S^{2}$ surfaces as leaves. It is constructed by taking the intersection of the null cones of the optical foliation, the $C_{u}$ 's, and the spatial hypersurfaces, $\Sigma$ 's, which are the leaves of the maximal foliation.

function $u$ defined in the entire region of space-time that is far from all mass. Thus, $u$ is the uniquely defined function that satisfies the so-called Eikonal equation:

$$
g^{\mu \nu} \frac{\partial u}{\partial x^{\mu}} \frac{\partial u}{\partial x^{\nu}}=0
$$

(d) Finally, to create the optical-maximal foliation we take the intersection of a given $C_{u}$ with a maximal hypersurface $\Sigma_{t}$ to get a topological $S^{2}$ surface which we name $S_{t, u}$. This foliation is shown in Fig. 3. We can always choose $t$ as big as needed to get the desired "closeness" to null infinity. [Taking this intersection is the same as using $l^{t}, \underline{l}^{t 4}$ to propagate the $S_{t, u}$ foliation along each $C_{u}$. In this way, one could thus vary the foliation by picking other null pairs (cf. Appendixes $\mathrm{C}$ and $\mathrm{D}$ ); we shall have occasion in this paper to do this.]

In order to understand the reason for picking this foliation we first introduce the ADM definition.

The ADM definition of angular momentum on a leaf $\Sigma_{t}$ has the form

$$
L_{s}^{(i)}(t)=\frac{1}{8 \pi} \lim _{r \rightarrow \infty} \int_{S_{r}} \varepsilon_{i a b} x^{a}\left(k^{b j}-g^{b j} \operatorname{tr} k\right) N_{j} d A
$$

where $S_{r}$ coordinate sphere of radius $r \sim \sqrt{\sum_{i} x_{i}^{2}}, x^{i}$ s defined in neighborhood of spatial infinity, $k^{i j}$ is the extrinsic curvature of the leaf $\Sigma_{t}, N$ is exterior unit normal to $S_{r}$, and $d A$ is an area element of $S_{r}$.

Equation (2) can be re-written, given a strongly asymptotically flat data set (cf. Appendix A):

\footnotetext{
${ }^{4}$ If we use $9.14 \mathrm{c}$ on p. 264 of [8] (note $\phi$ and $a$ are given in construction of maximal foliation), we obtain $l^{t}=\phi a l$.
} 


$$
\begin{aligned}
L_{s}^{(i)}(t) & =\frac{1}{8 \pi} \lim _{r \rightarrow \infty} \int_{S_{r}}\left\{\varepsilon_{i a b} x^{a} k^{b j} N_{j}-\varepsilon_{i a b} x^{a} N^{b} \operatorname{tr} k\right\} d A \\
& =\frac{1}{8 \pi} \lim _{r \rightarrow \infty} \int_{S_{r}} \varepsilon_{i a b} x^{a} k^{b j} N_{j} d A \\
& =\frac{1}{8 \pi} \lim _{r \rightarrow \infty} \int_{S_{r}} k_{A N} \Omega_{(i)}^{A} d A \\
& =\frac{1}{8 \pi} \lim _{\substack{t=\text { const } \\
u \rightarrow-\infty}} \int_{S_{r}} \epsilon_{A} \Omega_{(i)}^{A} d A
\end{aligned}
$$

where $\epsilon_{A} \equiv k_{A N}=\frac{1}{2}\left\langle D_{A} l^{\prime}, \underline{l}^{\prime}\right\rangle, \quad l^{\prime}=T+N, \quad \underline{l}^{\prime}=T-N$,

$$
\text { with } l=a^{-1} l^{\prime}, \quad \underline{l}=a \underline{l}^{\prime} \Rightarrow \epsilon_{A}=W_{A}-\bigotimes_{A} \ln a,
$$

$$
W_{A}=\frac{1}{2}\left\langle D_{A} l, \underline{l}\right\rangle,
$$

$$
\text { also } l s=1, \underline{l} u=2, a^{-1}=-D_{T} u=|\nabla u|
$$

$\nabla_{A}$ means covariant derivative on the $S^{2}$ surface

$A \in\{1,2\}, \Omega_{(i)}^{A}$ is rotation vector field around the $\hat{\imath}$ axis.

$\Omega_{(i)}^{A}$ are such that $\left[\Omega_{(i)}, \Omega_{(j)}\right]=\varepsilon_{i j k} \Omega_{(k)}$. They are rotation vector fields (they are defined pictorially in [4,9]) defined on "pulled back" sphere's (cf. Appendix F). The rotation vector fields correspond, in a Euclidean space, to rotations around the $x, y$ and $z$ coordinate axes. We will propagate these fields from one pulled back sphere to another using Lie dragging in the most natural way. We start from null infinity on the appropriate $C_{u}$ where the spheres are actual two-spheres without need of pull-back (and so $\Omega_{(i)}^{A}$ are defined very intuitively) and drag them into the space-time using $l$ [which to a sufficient order, near null infinity, is the same as propagating using $l^{t}=a \phi l$ (see below)]. $l$ is the most natural because it is a tangent to a null geodesic which is the path taken by gravity waves.

In short, in terms of the parameters of the above opticalmaximal foliation, the ADM angular momentum is (using the surface $\Sigma_{t=0}$ )

$$
L_{A D M}^{(i)} \equiv L_{\text {spatial }}=\lim _{u \rightarrow-\infty} \frac{1}{8 \pi} \int_{S_{0, u}} \epsilon_{A} \Omega_{(i)}^{A} d \mu_{\gamma} .
$$

Here $d \mu_{\gamma}$ is the differential area element of $S_{0, u}$ and $\gamma$ is the metric on $S_{0, u}$. The null-AM definition takes the following form using the geodesic-null pair (cf. Appendixes $\mathrm{C}$ and D) $l, \underline{l}$ in the optical-maximal foliation:

$$
\begin{aligned}
L_{\text {null }}^{(i)}(u) & =\lim _{\substack{u=\text { const } \\
s \rightarrow \infty}} \frac{1}{8 \pi} \int_{S_{e, u}} W_{A} \Omega_{(i)}^{A} d \mu_{\gamma} \\
& =\frac{1}{8 \pi} \int_{S_{u}}\left(Z_{A}^{(2)}-Z_{A} H\right) \Omega_{(i)}^{A} d \mu_{\gamma^{0}} .
\end{aligned}
$$

Here $S_{u} \equiv S_{\infty, u}$ is the $u$ element of the foliation of null infinity, and $d \mu_{\gamma^{0}}$ refers to the area element of $S_{u}$. The definition of the other quantities are given in Appendixes B and C. Equation (9) is the same as the definition in [4], because the geodesic-null pair manifestly (cf. Appendix D) reduces to the affine foliation [4] near null infinity.

Appendix B, in turn, shows that the limit using the geodesic-null pair to foliate the null cones is the same as using the $t$-null pair (cf. Appendixes C and D) $l^{t}, \underline{l}^{t}$. Thus, we have

$$
L_{\text {null }}^{(i)}(u)=\lim _{\substack{u=\text { const } \\ t \rightarrow \infty}} \frac{1}{8 \pi} \int_{S_{t, u}} W_{A} \Omega_{(i)}^{A} d \mu_{\gamma}^{t} .
$$

Now, to make the above somewhat fuzzy postulate, 1, more precise we take

$$
L_{t, u}^{(i)}(u)=\frac{1}{8 \pi} \int_{S_{t, u}} \epsilon_{A} \Omega_{(i)}^{A} d \mu_{\gamma}^{t}
$$

where everything is again in the optical-maximal foliation using the $t$-null pair. For example, the area element is that of a small piece of $S_{t, u}$ called $d \mu_{\gamma}^{t}$. If we can show

$$
\lim _{u \rightarrow-\infty} L_{\text {null }}^{(i)}(u)=\lim _{u \rightarrow-\infty} \lim _{t \rightarrow \infty} L_{t, u}^{(i)}
$$

then we can rewrite Eq. (1) which we seek to prove:

$$
\begin{gathered}
L_{\text {spatial }}=\lim _{u \rightarrow-\infty} L_{\text {null }}^{(i)}(u) \\
\text { becomes } \\
\lim _{u \rightarrow-\infty} L_{t=0, u}^{(i)}=\lim _{u \rightarrow-\infty} \lim _{t \rightarrow \infty} L_{t, u}^{(i)} .
\end{gathered}
$$

Here, to be concrete, we set $t=0$; Eq. (12) is of course true for every constant $t$ slice.

[Note using the fact the ADM angular momentum is the same on every time slice, one can write Eq. (12) as:

$$
\lim _{t \rightarrow \infty} \lim _{u \rightarrow-\infty} L_{t, u}^{(i)}=\lim _{u \rightarrow-\infty} \lim _{t \rightarrow \infty} L_{t, u}^{(i)} .
$$

That is, under the stated conditions, one can swap the $u$ and $t$ limits.]

There are thus two equations that must be proved: Eq. (10) and Eq. (12). We now proceed to those proofs; we prove Eq. (12) in Secs. II and III, and Eq. (10) is proved in Sec. IV. 


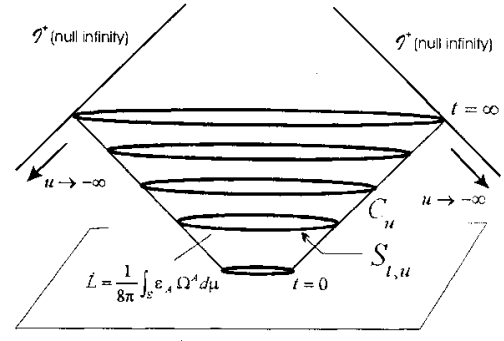

FIG. 4. Pictorial of LHS of inequality (14). For each surface $S_{t, u}$ one does the $\dot{L}$ integral shown. One then integrates $\dot{L}$ over $C_{u}$ on the region from $t=0$ to $t=\infty$. Finally, one takes the limit of such integrals as $u \rightarrow-\infty$.

\section{PROOF THAT $\lim _{u \rightarrow-\infty} L_{0, u}^{(i)}=\lim _{u \rightarrow-\infty} \lim _{t \rightarrow \infty} L_{t, u}^{(i)}$ WHEN $|\dot{L}|<C / r^{\beta}$ FOR $\beta>1$}

Because $L_{0, u}^{(i)}$ is independent of $t$, one can insert the $t$ limit (i.e. $\left.L_{0, u}^{(i)}=\lim _{t \rightarrow \infty} L_{0, u}^{(i)}\right)$ on the LHS of Eq. (12), and rewrite it as

$$
\begin{aligned}
\lim _{u \rightarrow-\infty} \lim _{t \rightarrow \infty}\left\{L_{0, u}^{(i)}-L_{t, u}^{(i)}\right\} & =0 \\
\Delta^{\prime} & =\lim _{u \rightarrow-\infty}\left\{L_{0, u}^{(i)}-L_{\infty, u}^{(i)}\right\}=0 .
\end{aligned}
$$

Hence, we want to show that $\Delta^{\prime}=0$.

Using the fundamental theorem of calculus, one obtains

$$
\Delta^{\prime} \equiv \lim _{u \rightarrow-\infty} \lim _{t \rightarrow \infty} \int_{0}^{t} \frac{\partial L\left(t^{\prime}, u\right)}{\partial t} d t^{\prime}
$$

We further define

$$
\Delta \equiv \lim _{u \rightarrow-\infty} \lim _{t \rightarrow \infty} \int_{0}^{t}\left|\frac{\partial L\left(t^{\prime}, u\right)}{\partial t}\right| d t^{\prime} .
$$

It is easy to show that

$$
\Delta=0 \Rightarrow \Delta^{\prime}=0 .
$$

We will now show that $\Delta=0$ if

$$
\left|\frac{\partial L(t, u)}{\partial t}\right|=|\dot{L}|<\frac{C}{r^{\beta}}
$$

for all $\beta>1$ and where $C$ is a constant

$$
\lim _{u \rightarrow-\infty} \int_{0}^{\infty}|\dot{L}| d t<\lim _{u \rightarrow-\infty} \int_{0}^{\infty} \frac{C}{r^{\beta}} d t .
$$

The limit on the LHS of Eq. (14) is illustrated in Fig. 4. The integrand, $|\dot{L}|$, in this limit is an integral on an $S_{t, u}$, an $S^{2}$ surface (after pull back). One integrates $|\dot{L}|$ along the null cone $C_{u}$ from $t=0$ out to null infinity, and then takes the limit of such integrals towards spatial infinity $i^{0}$. To evaluate the RHS of Eq. (14), we need to rewrite $d t$ in terms of $d r$. For a given $S_{t, u}$, one defines the areal radius, $r$, in the following way:

$$
A(t)=4 \pi r^{2}=\int d \mu_{\gamma}^{t}
$$

where $d \mu_{\gamma}^{t}$ is the area element of the $S^{2}$ surface of constant $t$ and on a given constant- $u$ cone

$$
\begin{gathered}
\frac{\partial}{\partial t} A=8 \pi r \frac{\partial r}{\partial t}=\int \operatorname{tr} \chi^{t} d \mu_{\gamma}^{t} \\
\Rightarrow \frac{\partial r}{\partial t}=\frac{r}{2} \overline{\operatorname{tr} \chi^{t}},
\end{gathered}
$$

where the overbar means averaged over solid angle. Using the decay law for $\operatorname{tr} \chi^{t}$ given in Appendix D

$$
\begin{aligned}
& \overline{\operatorname{tr} \chi^{t}} \sim \frac{2}{r}+\frac{\overline{H^{t}}}{r^{2}} \\
& \Rightarrow \frac{\partial r}{\partial t} \sim 1+\frac{\overline{H^{t}}}{2 r} \\
& \Rightarrow d t=\left(1-O\left(r^{-1}\right)\right) d r .
\end{aligned}
$$

Therefore we have

$$
\begin{aligned}
\lim _{u \rightarrow-\infty} \int_{0}^{\infty} \frac{C}{r^{\beta}} d t & =\lim _{u \rightarrow-\infty} \int_{0}^{\infty} \frac{C}{r^{\beta}}\left(1-O\left(r^{-1}\right)\right) d r \\
& =\lim _{u \rightarrow-\infty}\left(\int_{r_{0}}^{\infty} \frac{C}{r^{\beta}} d r-\int_{r_{0}}^{\infty} O\left(r^{-1-\beta}\right) d r\right) \\
& =\lim _{u \rightarrow-\infty}\left(\int_{r_{0}}^{\infty} \frac{C}{r^{\beta}} d r-\left[O\left(r^{-\beta}\right)\right]_{r_{0}}^{\infty}\right) \\
& =\lim _{u \rightarrow-\infty} \int_{r_{0}}^{\infty} C r^{-\beta} d r \\
& =C \lim _{r_{0} \rightarrow \infty}\left[\frac{r^{-\beta+1}}{-\beta+1}\right]_{r_{0}}^{\infty}
\end{aligned}
$$

where we use the fact that $r_{0} \rightarrow \infty$ as $u \rightarrow-\infty$. Now, the RHS of the last equality is zero as long as $\beta>1$. Since $\Delta$ $=0 \Rightarrow \Delta^{\prime}=0$, we have

$$
\lim _{u \rightarrow-\infty} L_{0, u}^{(i)}=\lim _{u \rightarrow-\infty} \lim _{t \rightarrow \infty} L_{t, u}^{(i)}
$$

Q.E.D.

We must now show that the condition given in Eq. (13) holds. 


\section{FALL OFF OF $\dot{L}$ FOR LARGE $r$}

We now proceed to show that $\dot{L}$ falls off like $C / r^{1+\varepsilon}$ for $\varepsilon>0$ where $C$ is a constant. In more specific terms, at a given point of space-time $\dot{L}$ will be associated with a unique leaf of the foliation, say $S_{t, u}$, which has areal radius $r$. As long as $r$ is sufficiently large (i.e. one is sufficiently far from the source), we will see that $\dot{L}$ decays like $1 / r^{1+\varepsilon}$ independent of $t$ or $u$ :

$$
\begin{aligned}
\dot{L} \equiv \frac{\partial L(t, u)}{\partial t}= & \frac{\partial}{\partial t} \int_{S_{t, u}} \epsilon_{A} \Omega^{A} d \mu_{\gamma}^{t}=£_{l^{t}} \int_{S_{t, u}} \epsilon_{A} \Omega^{A} d \mu_{\gamma}^{t} \\
= & \int_{S_{t, u}} £_{l^{t}}\left(\epsilon_{A}\right) \Omega^{A} d \mu_{\gamma}^{t}+\int_{S_{t, u}} \epsilon_{A} £_{l^{\prime}} \Omega^{A} d \mu_{\gamma}^{t} \\
& +\int_{S_{t, u}} \epsilon_{A} \Omega^{A} \operatorname{tr} \chi^{t} d \mu_{\gamma}^{t} \\
= & \int_{S_{t, u}} £_{l^{t}}\left(\epsilon_{A}\right) \Omega^{A} d \mu_{\gamma}^{t} \\
& +\int_{S_{t, u}} \epsilon_{A} \operatorname{tr} \chi^{t} \Omega^{A} d \mu_{\gamma}^{t} .
\end{aligned}
$$

The second equality, makes use of $\left(f_{l^{t}} d \mu_{\gamma}^{t}\right)=\operatorname{tr} \chi^{t} d \mu_{\gamma}^{t}$, and the third equality utilizes the fact that the rotation vector fields are moved forward according to the rule $£_{l} \Omega^{A}$ $=\left[l^{t}, \Omega^{A}\right]=0$. We will need the following:

$$
\begin{aligned}
£_{l^{t} V_{A}} & =\left(\left[l^{t}, V\right], e_{A}\right) \\
& =\left(D_{l^{t}} V, e_{A}\right)-\left(D_{V} l^{t}, e_{A}\right) \\
& =D_{l^{t}} V_{A}-V_{B}\left(D_{B} l^{t}, e_{A}\right) \\
& =D_{l^{t}} V_{A}-V_{B} \cdot \chi_{A}^{t B}
\end{aligned}
$$

where $V$ means any vector. Recalling $l^{t}=\phi a l$, we obtain

$$
\begin{aligned}
£_{l^{t}} V_{A} & =\mathbb{D}_{l^{t}} V_{A}-V_{B} \cdot \chi^{t B}{ }_{A} \\
& =\phi a D_{l} V_{A}-\phi a V_{B} \cdot \chi^{t B}{ }_{A} \\
& =\phi a £_{l} V_{A} .
\end{aligned}
$$

In the last line, use is made of the first line with $l$ substituted for $l^{t}$. Hence Eq. (16) becomes

$$
\begin{aligned}
= & \int_{S_{t, u}} \phi a\left(£_{l}\left(\epsilon_{A}\right)+\epsilon_{A} \operatorname{tr} \chi\right) \Omega^{A} d \mu_{\gamma}^{t} \\
= & \int_{S_{t, u}} \phi a\left(£_{l}\left(W_{A}-\bigotimes_{A} \ln a\right)\right. \\
& \left.+\left(W_{A}-\bigotimes_{A} \ln a\right) \operatorname{tr} \chi\right) \Omega^{A} d \mu_{\gamma}^{t} .
\end{aligned}
$$

To show the decay law of the integral above, we now need the decay laws for the each of the quantities in the integrand. The decay laws for the Ricci Coefficients are given in Appendix $\mathrm{D}$; we also need $\ln a \sim \Psi^{\prime} / r$ and $\ln \phi$ $\sim \Psi / r$ (all from [8]). These decay laws are valid for $t \rightarrow \infty$ on a constant $u$ cone. However, it can be shown that the decay laws in terms of the luminosity radius, $r$, fall off the same for $r \rightarrow \infty, t=$ const (confer Appendix E). Hence, substituting the decay laws into Eq. (21) above we obtain

$$
\begin{aligned}
& \int_{S_{t, u}}\left(1+\frac{\Psi}{r}\right)\left(1+\frac{\Psi}{r}\right)(\cdots) \Omega^{A}\left(r^{2}-r H^{t}\right) d \mu_{\gamma}^{0} \\
& =\int_{S_{t, u}}\left(\begin{array}{c}
£_{l}\left(\frac{Z_{A}-\bigotimes_{A} \Psi^{\prime}}{r}+\frac{Z_{A}^{(2)}-\bigotimes_{t} \Psi^{2}}{r^{2}}\right) \\
+\left(\frac{Z_{A}-\bigotimes_{A} \Psi^{\prime}}{r}+\frac{Z_{A}^{(2)}-\bigotimes_{A} \Psi^{(2)}}{r^{2}}\right)\left(\frac{2}{r}+\frac{H}{r^{2}}\right)
\end{array}\right)\left(r^{2}-r\left[H^{t}-\left(\Psi+\Psi^{\prime}\right)\right]\right) \Omega^{A} d \mu_{\gamma}^{0} \\
& =\int_{S_{t, u}}\left(\begin{array}{c}
-\frac{Z_{A}-\bigotimes_{A} \Psi^{\prime}}{r^{2}} £_{1} r-2 \frac{Z_{A}^{(2)}-\bigotimes_{A} \Psi^{(2)}}{r^{3}} £_{l} r \\
+2 \frac{Z_{A}-\bigotimes_{A} \Psi^{\prime}}{r^{2}}+2 \frac{Z_{A}^{(2)}-\bigotimes_{A} \Psi^{(2)}}{r^{3}}+\frac{H\left(Z_{A}-\bigotimes_{A} \Psi^{\prime}\right)}{r^{3}}
\end{array}\right)(\cdots) \Omega^{A} d \mu_{\gamma}^{0} \\
& =\int_{S_{t, u}}\left(\begin{array}{c}
-\frac{Z_{A}-\bigotimes_{A} \Psi^{\prime}}{r^{2}}-2 \frac{Z_{A}^{(2)}-\bigotimes_{A} \Psi^{(2)}}{r^{3}} \\
+2 \frac{Z_{A}-\bigotimes_{A} \Psi^{\prime}}{r^{2}}+2 \frac{Z_{A}^{(2)}-\bigotimes_{A} \Psi^{(2)}}{r^{3}}+\frac{H\left(Z_{A}-\bigotimes_{A} \Psi^{\prime}\right)}{r^{3}}
\end{array}\right)(\cdots) \Omega^{A} d \mu_{\gamma}^{0}
\end{aligned}
$$




$$
\begin{aligned}
& =\int_{S_{t, u}}\left(-\frac{Z_{A}-\bigvee_{A} \Psi^{\prime}}{r^{2}}+2 \frac{Z_{A}-\bigvee_{A} \Psi^{\prime}}{r^{2}}+\frac{H\left(Z_{A}-\bigvee_{A} \Psi^{\prime}\right)}{r^{3}}\right)\left(r^{2}-r(H+k)\right) \Omega^{A} d \mu_{\gamma}^{0} \\
& =\int_{S_{t, u}}\left(-(H+k) \frac{\left(Z_{A}-\bigvee_{A} \Psi^{\prime}\right)}{r}+H \frac{Z_{A}-\bigvee_{A} \Psi^{\prime}}{r}+O\left(r^{-(1+\epsilon)}\right)\right) \Omega^{A} d \mu_{\gamma}^{0} \\
& =O\left(r^{-(1+\epsilon)}\right) \\
& \Rightarrow \dot{L} \sim O\left(r^{-(1+\epsilon)}\right) \quad \text { Q.E.D. }
\end{aligned}
$$

In the above, ${ }^{5}$ use is made of $£_{l} r=(\partial r / \partial s)=a^{-1} \phi^{-1}(\partial r / \partial t) \sim\left[1-\left(\Psi+\Psi^{\prime}\right) / r\right]\left(1+\bar{H}^{t} / 2 r\right)=1+\mathrm{const} / r$. $\int_{S_{t, u}} Z_{A} \Omega^{A} d \mu_{\gamma^{0}}=0$ and $\int_{S_{t, u}} \downarrow_{A} \Psi^{\prime} \Omega^{A} d \mu_{\gamma^{0}}=0$ (proved in Appendix B)

$$
H^{t}=H+2\left(\Psi+\Psi^{\prime}\right)
$$

with $\left(\Psi+\Psi^{\prime}\right)=$ const $\equiv k$ (using p. $\left.504[8]\right)$.

\section{PROOF THAT $\lim _{u \rightarrow-\infty} L_{\text {null }}^{(i)}(u)=\lim _{u \rightarrow-\infty} \lim _{t \rightarrow \infty} L_{t, u}^{(i)}$}

$$
\begin{aligned}
& \lim _{u \rightarrow-\infty t \rightarrow \infty} \lim _{t, u}^{(i)}=\frac{1}{8 \pi} \lim _{u \rightarrow-\infty t \rightarrow \infty} \int_{S_{t, u}} \epsilon_{A} \Omega_{(i)}^{A} d \mu_{\gamma}^{t} \\
& =\frac{1}{8 \pi} \lim _{u \rightarrow-\infty t \rightarrow \infty} \int_{S_{t, u}}\left(W_{A}-\not_{A} \ln a\right) \Omega_{(i)}^{A} d \mu_{\gamma}^{t} \\
& =\frac{1}{8 \pi} \lim _{u \rightarrow-\infty r \rightarrow \infty} \lim _{S_{t, u}}\left(\frac{Z_{A}^{(2)}-\nabla_{A} \Psi^{(2)}}{r^{2}}+\frac{Z_{A}-\bigotimes_{A} \Psi^{\prime}}{r}\right)\left(r^{2}-r H^{t}\right) \Omega_{(i)}^{A} d \mu_{\gamma} 0 \\
& =\frac{1}{8 \pi} \lim _{u \rightarrow-\infty r \rightarrow \infty} \lim _{S_{t, u}}\left(\frac{Z_{A}^{(2)}-\downarrow_{A} \Psi^{(2)}}{r^{2}}+\frac{Z_{A}-\nabla_{A} \Psi^{\prime}}{r}\right)\left(r^{2}-r H^{t}\right) \Omega_{(i)}^{A} d \mu_{\gamma^{0}} \\
& =\frac{1}{8 \pi} \lim _{u \rightarrow-\infty} \int_{S_{t, u}}\left(Z_{A}^{(2)}-Z_{A} H+H \bigotimes_{A} \Psi^{\prime}\right) \Omega_{(i)}^{A} d \mu_{\gamma^{0}} \\
& =\frac{1}{8 \pi} \lim _{u \rightarrow-\infty}\left(L_{n u l l}^{(i)}(u)+\int_{S_{t, u}} H \bigotimes_{A} \Psi^{\prime} \Omega_{(i)}^{A} d \mu_{\gamma^{0}}\right)
\end{aligned}
$$

where $H^{t}=$ const $+H$.

In the penultimate equality, use is made of $\int_{S_{\infty, u}} Z_{A} \Omega_{(i)}^{A} d \mu_{\gamma^{0}}=0$. In the last equality, one uses Eq. (9). Finally, in order to complete the proof, we must show that

$$
\lim _{u \rightarrow-\infty} \int_{S_{t, u}} H \bigotimes_{A} \Psi^{\prime} \Omega_{(i)}^{A} d \mu_{\gamma^{0}}=0
$$

or

${ }^{5}$ It is interesting to note that the final decay law given above would also apply in the more general case: $\ln a \sim 1+\Psi^{\prime} / r$ $+O\left(r^{-(1+\epsilon)}\right)$.

$$
\int_{S_{t, u}} H^{-} \bigotimes_{A} \Psi^{\prime-} \Omega_{(i)}^{A} d \mu_{\gamma^{0}}=0 .
$$

We will designate the LHS of Eq. (23) by $\star$. To evaluate the integral $\star$ we must find expressions for $H^{-}$and $\Psi^{\prime-}$; we will find the expressions in the given order.

\section{A. Expression for $\boldsymbol{H}^{-}$}

Using an equation on p. 508 of [8] and $Z=E+\not \Psi^{\prime}$ [note that the $u$ (the retarded time) and $\Xi$ (null ingoing sheer) that I use below are related to the $u$ and $\Xi$ of Christodoulou and Klainerman (CK) [8] in the following way: $u_{C K}=-u$ and $\Xi_{C K}=-1 / 2 \Xi$; the minus superscript on a quantity means $u \rightarrow-\infty]$ 


$$
\begin{aligned}
(\nabla \cdot Z)^{-}= & \left(\Delta \Psi^{\prime}-\frac{1}{2} \Sigma \cdot \Xi+\frac{1}{2} \overline{\Sigma \cdot \Xi}+P-\bar{P}\right. \\
& \left.+\Delta \Psi-\Psi^{\prime}-\Omega^{\prime}\right)^{-}
\end{aligned}
$$

Now using $(P-\bar{P})^{-}=0, \Xi^{-}=0$, and $Z=\nabla \cdot \Sigma-1 / 2 \not H$ one obtains

$$
\nabla \cdot\left(\nabla \cdot \Sigma-\frac{1}{2} \not H\right)^{-}=\Delta\left(\Psi^{\prime}-\Psi\right)^{-}-\left(\Psi^{\prime}+\Omega^{\prime}\right)^{-} .
$$

In the above, the overbar means averaged over $S^{2}$.

Next we use the gauge condition (from [4]) that the odd part of the sheer is zero; mathematically, one can the write

$$
\nabla_{B} \Sigma^{B A}=\varepsilon_{A B} \not^{B} \phi_{\text {even }} \Rightarrow \Delta \Sigma=0 .
$$

Hence, recalling that $\Psi^{\prime}+\Psi=$ const:

$$
\begin{aligned}
-\frac{1}{2} \Delta H^{-} & =-\left(\Psi^{\prime}+\Omega^{\prime}\right)^{-} \Rightarrow \\
\Delta H^{-} & =2\left(\Psi^{\prime}+\Omega^{\prime}\right)^{-} \\
& =f(\omega) .
\end{aligned}
$$

Now, expanding $H$ and $f$ in spherical harmonics (where $\omega \in S^{2}$ e.g. $\left.\theta, \phi\right)$, e.g. $H^{-}=\Sigma_{l, m} H_{l, m}^{-} Y_{l, m}(\omega)$, implies that Eq. (26) becomes

$$
\begin{gathered}
\sum_{l, m} l(l+1) H_{l, m}^{-} Y_{l, m}(\omega)=-\sum_{l, m} f_{l m} Y_{l, m}(\omega) \\
\Rightarrow \\
H^{-}=-\sum_{l, m} \frac{1}{l(l+1)} f_{l, m} Y_{t, m}(\omega) .
\end{gathered}
$$

Now, we must find the harmonic coefficients $f_{l, m}$ that characterize $2\left(\Psi^{\prime}+\Omega^{\prime}\right)^{-}$. Rewriting the solution for these quantities on p. 504 of [8], one gets

$$
\begin{aligned}
\left(\Psi^{\prime}+\Omega^{\prime}\right)^{-}= & \frac{1}{16 \pi}\left[\int_{-\infty}^{\infty} d u^{\prime} \int_{S^{2}} \frac{\left(|\Xi|^{2}\left(u^{\prime}, \vec{x}\right)-\mid \overline{\Xi^{2}\left(u^{\prime}\right) \mid}\right)}{\left|\vec{x}-\vec{x}^{\prime}\right|} d \mu_{\left(x^{\prime}\right)}(1-1 / 2)\right] \\
& -\frac{1}{8} \int_{-\infty}^{\infty} d u^{\prime} \operatorname{sgn}\left(\infty+u^{\prime}\right)\left(|\Xi|^{2}\left(u^{\prime}, \omega\right)-\left|\Xi^{2}\left(u^{\prime}\right)\right|\right)
\end{aligned}
$$

where the overbar means average over $S^{2}$, and $d \mu_{\left(x^{\prime}\right)}$ is the differential area element of $S^{2}$ in terms of the primed coordinates, $x^{\prime}$. Note that $\operatorname{sgn}\left(\infty+u^{\prime}\right)=+1$ and $\partial M(u) / \partial u=1 / 32 \pi \int\left|\Xi^{2}\left(u^{\prime}\right)\right| d \mu_{\gamma}^{0}$ gives $\int_{-\infty}^{\infty} \overline{\left|\Xi^{2}\left(u^{\prime}\right)\right|} d u^{\prime}=8\left(M_{f}-M_{i}\right)=8 \Delta M$ where $M_{f} \equiv M(+\infty)$ and $M(-\infty)=M_{i}$. Note also that we have picked arbitrary $z$ axis and defined the coordinates $\theta$, $\varphi$ (written abbreviated as $\omega$ ) in usual way. We also need

$$
\begin{aligned}
\frac{1}{|\vec{x}-\vec{x}|}= & \sum_{l=0}^{\infty} \sum_{m=-l}^{m=l} \frac{4 \pi}{2 l+1} Y_{l m}^{*}\left(\theta^{\prime}, \varphi^{\prime}\right) Y_{l m}(\theta, \varphi) \\
& \left(\text { using }|\vec{x}|=\left|\vec{x}^{\prime}\right|=1\right) \\
\int_{S^{2}} \frac{1}{\left|\vec{x}-\vec{x}^{\prime}\right|} d \mu_{\left(x^{\prime}\right)}= & \int \frac{\sqrt{4 \pi} Y_{00}\left(\theta^{\prime}, \varphi^{\prime}\right)}{|\vec{x}-\vec{x}|} d \mu_{\left(x^{\prime}\right)} \\
= & 4 \pi Y_{00}(\theta, \varphi) \int Y_{00}^{*}\left(\theta^{\prime}, \varphi^{\prime}\right) \sqrt{4 \pi} Y_{00}\left(\theta^{\prime}, \varphi^{\prime}\right) d \omega^{\prime} \\
= & 4 \pi
\end{aligned}
$$

where $d \omega^{\prime}=\sin \theta d \theta d \phi$ and using $\widetilde{F}(\omega) \equiv 8 S \equiv \int_{-\infty}^{\infty} d u^{\prime}\left|\Xi\left(u^{\prime}, \omega\right)\right|^{2}=\int_{-\infty}^{\infty} d u^{\prime}\left|\Xi\left(u^{\prime}, \theta, \phi\right)\right|^{2}[S / 4 \pi=$ the total energy radiated (in the proper sense) per unit solid angle emitted in the direction $(\theta, \phi)[10]]$ we get

$$
\begin{aligned}
\left(\Psi^{\prime}+\Omega^{\prime}\right)^{-} & =\frac{1}{2}\left(\frac{1}{16 \pi} \int_{S^{2}} \frac{\widetilde{F}\left(\vec{x}^{\prime}\right)}{\left|\vec{x}-\vec{x}^{\prime}\right|} d \mu_{\left(x^{\prime}\right)}-2 \Delta M\right)-\frac{1}{8}(\widetilde{F}(\omega)-8 \Delta M) \\
& =\frac{1}{2} \frac{1}{16 \pi} \int_{S^{2}\left|\vec{x}-\vec{x}^{\prime}\right|}^{\widetilde{x}\left(\vec{x}^{\prime}\right)} d \mu_{\left(x^{\prime}\right)}-\frac{1}{8} \widetilde{F}\left(\omega^{\prime}\right) \\
& =\frac{1}{2} \frac{1}{16 \pi} \int \sum_{l, m} F_{l, m} Y_{l m}\left(\omega^{\prime}\right) \sum_{l^{\prime}, m^{\prime}} \frac{4 \pi}{2 l^{\prime}+1} Y_{l^{\prime} m^{\prime}}^{*}\left(\omega^{\prime}\right) Y_{l^{\prime}, m^{\prime}}(\omega) d \omega^{\prime}-\frac{1}{8} \sum_{l, m} F_{l, m} Y_{l, m}(\omega) \\
& =\frac{1}{2} \frac{1}{4} \sum_{l, m} \frac{F_{l, m}}{2 l+1} Y_{l m}(\omega)-\frac{1}{8} \sum_{l, m} F_{l, m} Y_{l, m}(\omega) \\
& =-\frac{1}{8} \sum_{l, m} \frac{2 l}{2 l+1} F_{l, m} Y_{l, m}(\omega)
\end{aligned}
$$


where

$$
\widetilde{F}(\omega)=\sum_{l=0}^{\infty} \sum_{m=-l}^{m=l} F_{l, m} Y_{l, m}(\theta, \varphi) .
$$

Finally, using Eqs. (25)-(27) we get

$$
H^{-}=\frac{1}{2} \sum_{l, m} \frac{1}{l(l+1)} \frac{l}{2 l+1} F_{l, m} Y_{l, m}(\omega) .
$$

\section{B. Expression for $\Psi^{\prime-}$}

In the manner above one gets

$$
\Psi^{\prime-}(\omega)=\frac{1}{4} \sum_{l, m} \frac{F_{l, m}}{2 l+1} Y_{l m}(\omega)-2 \Delta M
$$

Because of the form of Eq. (23), we can drop the $-2 \Delta M$ and, thus, for our purposes

$$
\Psi^{\prime-}=\frac{1}{4} \sum_{l, m} \frac{F_{l, m}}{2 l+1} Y_{l m}(\omega) .
$$

Since $\star$, the LHS of Eq. (23), is an integral on a sphere we can rewrite that integral as

$$
\star=-\int \Psi^{\prime-} \ngtr_{A} H^{-} \Omega_{(i)}^{A} d \mu_{\gamma^{0}}
$$

\section{Symmetry makes $\star=0$}

Therefore, if we pick, as an example, $\Omega_{(i)}^{A}=\Omega_{(z)}^{A}$ then $\nabla_{\Omega} H^{-}=L_{z} H^{-}$(with $L_{z}=\partial / \partial \phi$ and $L_{z} Y_{l m}=i m Y_{l m}$ ) and using Eqs. (28) and (30) one obtains

$$
\begin{aligned}
\star & =-\int \Psi^{\prime} L_{z} H^{-} d \mu_{\gamma^{0}} \\
& =\frac{1}{4} \int\left(\sum_{l, m} \frac{F_{l, m}}{2 l+1} Y_{l m}(\omega) L_{z}\left(-\frac{1}{2} \sum_{l^{\prime}=0}^{\infty} \sum_{m^{\prime}=-l^{\prime}}^{m^{\prime}=l^{\prime}} \frac{1}{l^{\prime}\left(l^{\prime}+1\right)} \frac{l}{2 l^{\prime}+1} F_{l^{\prime}, m^{\prime}} Y_{l^{\prime}, m^{\prime}}(\omega)\right)\right) d \mu_{\gamma^{0}} \\
& =-\frac{i}{8} \int \sum_{l, m} \frac{F_{l, m}}{2 l+l} Y_{l m}(\omega) \sum_{l^{\prime}=0}^{\infty} \sum_{m^{\prime}=-l}^{m=l} \frac{m^{\prime} F_{l^{\prime}, m^{\prime}}}{l^{\prime}\left(l^{\prime}+1\right)\left(2 l^{\prime}+1\right)} Y_{l^{\prime} m^{\prime}}(\omega) d \mu_{\gamma^{0} .} .
\end{aligned}
$$

Taking $m^{\prime} \rightarrow-m^{\prime}$ and using $Y_{l,-m}=Y_{l, m}^{*}$, one gets

$$
\begin{aligned}
\star & =-\frac{i}{8} \int \sum_{l, m} \frac{F_{l, m}}{2 l+l} Y_{l m}(\omega) \sum_{l^{\prime}=0}^{\infty} \sum_{m^{\prime}=-l}^{m^{\prime}=l^{\prime}} \frac{-m^{\prime} F_{l^{\prime},-m^{\prime}}}{\left(2 l^{\prime}+1\right) l^{\prime}\left(l^{\prime}+1\right)} Y_{l^{\prime} m^{\prime}}^{*}(\omega) d \mu_{\gamma^{0}} \\
& =-\frac{i}{8} \sum_{l=0}^{\infty} \sum_{m=-l}^{m=l} \frac{-m F_{l, m} F_{l,-m}}{(2 l+1)^{2} l(l+1)} \\
& =-\frac{i}{8}\left(\sum_{l=0}^{\infty} \frac{1}{(2 l+1)^{2} l(l+1)}\left(\sum_{m=1}^{l}-m F_{l, m} F_{l,-m}+\sum_{m=1}^{l} m F_{l, m} F_{l,-m}\right)\right)=0 .
\end{aligned}
$$


Since $z$ was picked arbitrarily, the same logic follows for $\Omega_{x}$ and $\Omega_{y}$ as well. Hence,

$$
\int_{S_{i, u}} H^{-} \bigotimes_{A} \Psi^{\prime-} \Omega_{(i)}^{A} d \mu^{0}=0 .
$$

Q.E.D.

What are the reasons this integral is zero? First, it would not, in general be zero without the gauge condition given for the null definition. It also relies on the invariance of the laplacian (both in two and three dimensions) under rotations and on the preservation of a certain symmetry due to the content of the integrand; it contains $H$ and $\Psi^{\prime}$, both of which are integrals of $\Xi^{2}$. Specifically, the invariance under rotation of the laplacian makes the kernels of the integrals of $\Xi^{2}$ rotation invariant (function of only distance). In terms of the spherical harmonic decomposition, one must have $F_{l, m}$ in both the $H^{-}$term and the $\Psi^{\prime-}$ terms with no other $m$ dependence; otherwise, the cancelation to give zero would not occur.

\section{CONCLUSION}

We have just shown that the definition of angular momentum given in [4], valid for all retarded time at null infinity, reduces to the ADM (spatial angular momentum) in the limit of retarded times infinitely far in the past. Mathematically, this is written $L_{\text {spatial (ADM) }}=\lim _{u \rightarrow-\infty} L_{n u l l}^{(i)}(u)$. The ADM angular momentum can be thought of as, in some sense, the "total' angular momentum of the space-time. The null-AM definition should yield this "total" before any radiation has had a chance to leave: that is, in the infinite past. Hence, the proof further solidifies the correctness of the new definition. Without the use of the gauge condition given in [4], and stated in Eq. (24) of this paper, the proof would fail.

One may now ask: why does the null definition require the gauge condition, while the spatial definition does not? At null infinity gravity waves are constantly changing and mixing the choices for $S^{2}$ surfaces of integration; one has to pick among the many possible choices. The gauge condition makes this choice. By contrast, the spatial definition requires no such choice, because it only gives the angular momentum in the infinite past ${ }^{6}$ and has no changing surfaces to deal with. Furthermore, in the case of the canonical ADM momentum $\left[P_{\mathrm{ADM}}=0\right.$ (the center of mass case) $]$ considered in this paper, one does not have to pick out the $S^{2}$ surfaces that correspond to position and thereby, for example, separate out the spin part from the orbital part. Since the $P \neq 0$ case does involve such a selection, it does require a gauge condition, but apparently for a different reason. To consider the $P_{\mathrm{ADM}}$ $\neq 0$ is an interesting and worthy project. It would involve deciding how to define the center of mass $S^{2}$ surfaces and would even further elucidate the issue of angular momentum in general relativity.

\footnotetext{
${ }^{6}$ Effectively, this means the spatial definition gives the (in some sense) "total" angular momentum for the space-time. This unfortunately means it can give no useful conservation law. That is, one cannot, using it, make the simple statement: a system had initial angular momentum $L$, and $\Delta L$ escaped leaving the system with $L$ $-\Delta L$. One can do this with the definition at null infinity.
}

Several other aspects of the proof given in this paper are worthy of note. First, the physical import of Eq. (23), specifically the symmetry issues and the functional dependence of $F$ is still to be explored and may hold significant insight. In a similar vein, it is interesting to simply note that the definition of angular momentum with the integrand $\epsilon_{A} \Omega^{A}$ and the definition using the integrand $W_{A} \Omega^{A}$ do not reduce to the same result at null infinity; this reveals something of the subtlety of angular momentum at null infinity. Lastly, the mathematics used to show the reduction is also interesting in its own right, because it is a powerful tool in working in the region far from all mass where gravity wave detectors operate.

\section{ACKNOWLEDGMENTS}

I would like to thank Demetrios Christodoulou for helpful conversation and for reading this manuscript for content. This work was supported in part by NSF Grants PHY9210038 and PHY98-01158.

\section{APPENDIX A: STRONGLY ASYMPTOTICALLY FLAT SPACE-TIMES}

Briefly, a strongly asymptotically flat space-time is one that can be constructed from an initial data set of the type described below.

An initial data set satisfies the condition of strongly asymptotically flatness (cf. [8]) if $g$ and $k$ (the metric and extrinsic curvature) are sufficiently smooth and there is a coordinate system $x^{i}$ such that as the radius $r \sim \sqrt{\sum_{i}\left(x^{i}\right)^{2}} \rightarrow \infty$

$$
\begin{aligned}
g_{i j} & =\left(1+\frac{2 M}{r}\right) \delta_{i j}+o_{4}\left(r^{-3 / 2}\right) \\
k_{i j} & =o_{3}\left(r^{-5 / 2}\right) .
\end{aligned}
$$

Note that a function is said to be $o_{m}\left(r^{-k}\right)$ as $r \rightarrow \infty$ if $\partial^{l} f(x)=o\left(r^{-k-l}\right) \forall l=\{0,1, \ldots m\}$ where $\partial^{l}$ denote all the partial derivatives of order $l$ relative to the coordinates $x^{1}, x^{2}, x^{3}$. The above conditions imply that the data set is taken in the center of mass frame $\left(P_{\mathrm{ADM}}=0\right)$.

\section{APPENDIX B: NULL DEFINITION CAN USE $S_{t, u}$ FOLIATION}

First, recall the null definition given in terms of the geodesic-null pair (or use the affine foliation [4,11] directly):

$$
L_{\text {null }}^{(i)}(u)=\lim _{\substack{u=\text { const } \\ s \rightarrow \infty}} \frac{1}{8 \pi} \int_{S_{s, u}} W_{A} \Omega_{(i)}^{A} d \mu_{\gamma} .
$$

Using

$$
\begin{aligned}
& W_{A} \sim Z_{A} / r+Z_{A}^{(2)} / r^{2}+\cdots \\
& \operatorname{tr} \chi \sim 2 / r+H / r^{2}+\cdots \\
& d \mu_{\gamma} \sim\left\{r^{2}-r(H-\bar{H})\right\} d \mu_{\gamma^{0}}
\end{aligned}
$$

(where superscript " 0 ', means area element on $S^{2}$ slice of null infinity) gives 


$$
\lim _{\substack{u=\text { const } \\ s \rightarrow \infty}} \frac{1}{8 \pi} \int_{S_{s, u}} W_{A} \Omega_{(i)}^{A} d \mu_{\gamma}=\frac{1}{8 \pi} \int_{S_{u}}\left(Z_{A}^{(2)}-Z_{A} H\right) \Omega_{(i)}^{A} d \mu_{\gamma^{0}}
$$

where use has been made of $\int_{S_{u}} Z_{A} \Omega_{(i)}^{A} d \mu_{\gamma^{0}}=0$. This is proven as follows:

$$
\begin{aligned}
\int_{S_{\infty, u}} Z_{A} \Omega_{(i)}^{A} d \mu_{\gamma^{0}} & =\int_{S_{\infty, u}}\left(\downarrow_{B} \Sigma_{A}^{B}-\frac{1}{2} \downarrow_{A} H\right) \Omega_{(i)}^{A} d \mu_{\gamma^{0}} \\
& =-\int_{S_{\infty, u}}\left(\Sigma_{A}^{B} \downarrow_{B} \Omega_{(i)}^{A}-\frac{1}{2} H \nabla_{A} \Omega_{(i)}^{A}\right) d \mu_{\gamma^{0}} \\
& =0
\end{aligned}
$$

because $\Omega_{(i)}^{A}$ is killing and $\Sigma^{B A}$ is symmetric. (Note: from the same argument, it is clear that $\int_{S_{\infty, u}} \downarrow_{A} \Psi^{\prime} \Omega_{(i)}^{A} d \mu_{\gamma^{0}}=0$.)

Now, using the $t$-null pair, in a precisely parallel way one gets

$$
\lim _{\substack{u=\text { const } \\ t \rightarrow \infty}} \frac{1}{8 \pi} \int_{S_{t, u}} W_{A} \Omega_{(i)}^{A} d \mu_{\gamma}^{t}=\frac{1}{8 \pi} \int_{S_{u}}\left(Z_{A}^{(2)}-Z_{A} H^{t}\right) \Omega_{(i)}^{A} d \mu_{\gamma^{0}} .
$$

But, as given in Eq. (24), $H^{t}=H+k$ where $k$ is a constant so one gets

$$
L_{\text {null }}^{(i)}(u)=\lim _{\substack{u=\text { const } \\ t \rightarrow \infty}} \frac{1}{8 \pi} \int_{S_{t, u}} W_{A} \Omega_{(i)}^{A} d \mu_{\gamma}
$$

If one uses the structure equations $h$ and $k$ given in [11] (originally from [8]) one can show the existence of this limit follows in same manner as for affine foliation. For the sake of completeness, the definition in terms of parameters that are in principle measurable is [4]

$$
L_{\text {null }}^{(i)}(u)=\frac{1}{8 \pi} \int\left(\Sigma_{A B} \phi_{C} \cdot \Sigma^{C B}+I_{A}\right) \Omega_{(i)}^{A} d \mu_{\gamma^{0}} .
$$

\section{APPENDIX C: RICCI ROTATION COEFFICIENTS}

The Ricci rotation coefficients are defined below with respect to a null pair, $e_{3}, e_{4}$ (where $e_{3}, e_{4}=-2$ and, in the
TABLE I. Standard null pair $\left(l^{\prime}, \underline{l}^{\prime}\right)$.

\begin{tabular}{ll}
\hline \hline$H_{A B}^{\prime}=\theta_{A B}-k_{A B}$ & $\underline{H}_{A B}^{\prime}=-\theta_{A B}-k_{A B}$ \\
$Z Z_{A}^{\prime}=\emptyset_{A} \ln a+\epsilon_{A}$ & $\underline{Z Z}_{A}^{\prime}=\downarrow_{A} \ln \phi-\epsilon_{A}$ \\
$Y_{A}^{\prime}=0$ & $\underline{Y}_{A}^{\prime}=\phi_{A} \ln \frac{\phi}{a}$ \\
$\Omega^{\prime}=\frac{1}{2}\left(-\nabla_{N} \ln \phi+\delta\right)$ & $\underline{\Omega}^{\prime}=\frac{1}{2}\left(\nabla_{N} \ln \phi+\delta\right)$ \\
$V_{A}^{\prime}=\epsilon_{A}=k_{A N}$ & \\
\hline \hline
\end{tabular}

case used most frequently in this paper, $e_{3}=\underline{l}^{t}, e_{4}=l^{t}$ ), and the spatial vectors, $e_{1}, e_{2}$, orthogonal to these two vectors and tangent to the topologically $S^{2}$ spheres which make up the foliation. The $e_{\mu}$ are called the null tetrads.

$$
\begin{aligned}
H_{A B} & =\left\langle D_{A} e_{4}, e_{B}\right\rangle, \quad \underline{H}_{A B}=\left\langle D_{A} e_{3}, e_{B}\right\rangle, \\
Z Z_{A} & =\frac{1}{2}\left\langle D_{3} e_{4}, e_{A}\right), \quad \underline{Z Z_{A}}=\frac{1}{2}\left\langle D_{4} e_{3}, e_{A}\right\rangle, \\
Y_{A} & =\frac{1}{2}\left\langle D_{4} e_{4}, e_{A}\right\rangle, \quad \underline{Y_{A}}=\frac{1}{2}\left\langle D_{3} e_{3}, e_{A}\right\rangle, \\
\Omega & =\frac{1}{4}\left\langle D_{4} e_{4}, e_{3}\right\rangle, \quad \underline{\Omega}=\frac{1}{4}\left\langle D_{3} e_{3}, e_{4}\right\rangle, \\
V_{A} & =\frac{1}{2}\left\langle D_{A} e_{4}, e_{3}\right\rangle .
\end{aligned}
$$

Note that the quantity $\Omega$ is not related to the quantity labeled $\Omega^{\prime}$ in Eq. (25).

The unsuperscripted null pair, $l, \underline{l}$ refer to the geodesic pair (so called because $l$ is tangent to a null geodesic); unsuperscripted Ricci rotation coefficients refer to those with respect to the geodesic null pairs $l, \underline{l}$. Superscript $t$ on the null pair refers to $l^{t}=\phi l, \underline{l}^{t}=\phi^{-1} \underline{l}$ (the $S^{2}$ foliation is propagated to be on a maximal hypersurface $t$ ). The primed null pair refers to the "standard" null pair: $l^{\prime}=T+N=a l^{\prime}, \underline{l}^{\prime}$ $=T-N=a^{-1} \underline{l}^{\prime}$ where $T$ is the unit normal to the maximal (spatial) hypersurface, and $N$ is the unit normal to $S_{t, u}$ in the maximal hypersurface. Ricci rotation coefficients associated with these null tetrads are distinguished respectively by superscript $t$ and primes.

For the standard null pair one gets (rearranging [8], p. 171) the results in Table I, where

$a=\frac{1}{|\nabla u|}=\left\{\right.$ the lapse function of the foliation induced by $u$ on each $\left.\Sigma_{t}\right\}$

$\theta_{A B}=\left\{\right.$ the extrinsic curvature of the surfaces $S_{t, u}$ relative to $\left.\Sigma_{t}\right\}$

$k_{i j}=\{$ extrinsic curvature of the maximal slice $\}$

$=\frac{1}{2}$ spatial components $\left(£_{T} \widetilde{g}_{i j}\right)=-(2 \phi)^{-1} \partial_{t} \widetilde{g}_{i j}$

Decomposition of $k: \eta_{A B}=k_{A B}, \quad \epsilon_{A}=k_{A N}, \quad \delta=k_{N N}$. 
TABLE II. Geodesic null pair $(l, \underline{l})$.

\begin{tabular}{|c|c|}
\hline$H_{A B}=a^{-1}\left(\theta_{A B}-k_{A B}\right)$ & $\underline{H}_{A B}=a\left(-\theta_{A B}-k_{A B}\right)$ \\
\hline$Z Z_{A}=\triangleright_{A} \ln a+\epsilon_{A}$ & $\underline{Z Z}_{A}=\nabla_{A} \ln \phi-\epsilon_{A}$ \\
\hline$Y_{A}=0$ & $\underline{Y}_{A}=a^{2}\left(\nabla_{A} \ln \frac{\phi}{a}\right)$ \\
\hline$\Omega=-a^{-1} \Omega^{\prime}+a^{-1} \Omega^{\prime}=0$ & $\underline{\Omega}=\frac{a}{2}\left(\nabla_{N} \ln \phi+\delta\right)-\frac{1}{2} D_{\underline{\underline{l}}^{\prime}} a$ \\
\hline$V_{A} \equiv W_{A}=\epsilon_{A}+\nabla_{A} \ln a$ & \\
\hline
\end{tabular}

One can transforms to a different null pair; this is called a lapse transformation ${ }^{7}$ and has the form

$$
l^{\text {Trans }}=a^{-1} l, \quad \underline{l}^{\text {Trans }}=a \underline{l}
$$

where $a$ is called the lapse function

and is any function on the $S^{2}$ surface.

Note that the normalization $l \cdot \underline{l}=-2$ is preserved under a lapse transformation. Under the lapse transformation above, the Ricci coefficients transform as

$$
\begin{aligned}
& H^{\text {Trans }}=a^{-1} H, \quad \underline{H}^{\text {Trans }}=a \underline{H}, \\
& Z Z^{\text {Trans }}=Z Z, \quad \underline{Z Z}^{\text {Trans }}=\underline{Z Z}, \\
& Y^{\text {Trans }}=a^{-2} Y, \quad \underline{Y}^{\text {Trans }}=a^{2} \underline{Y}, \\
& \Omega^{\text {Trans }}=\frac{1}{2} a^{-2} D_{l} a+a^{-1} \Omega, \quad \underline{\Omega}^{\text {Trans }}=a \underline{\Omega}-\frac{1}{2} D_{\underline{l}} a, \\
& V^{\text {Trans }}=V+\downarrow \ln a .
\end{aligned}
$$

TABLE III. t-null pair $\left(l^{t}, \underline{l}^{t}\right)$.

\begin{tabular}{ll}
\hline \hline$H_{A B}^{t}=\phi\left(\theta_{A B}-k_{A B}\right)$ & $\underline{H}_{A B}^{t}=\phi^{-1}\left(-\theta_{A B}-k_{A B}\right)$ \\
$Z Z_{A}^{t}=\bigotimes_{A} \ln a+\epsilon_{A}$ & $\underline{Z Z}_{A}^{t}=\bigotimes_{A} \ln \phi-\epsilon_{A}$ \\
$Y_{A}^{t}=0$ & $\underline{Y}_{A}^{t}=\phi^{-2}\left(\nabla_{A} \ln \frac{\phi}{a}\right)$ \\
$\Omega^{t}=\frac{1}{2} \phi^{2} D_{l^{\prime}} \phi^{-1}$ & $\underline{\Omega^{t}=\frac{\phi^{-1}}{2}\left(\nabla_{N} \ln \phi+\delta\right)-\frac{1}{2} D_{\underline{l}^{\prime}} \phi^{-1}}$ \\
$\quad+\frac{\phi}{2}\left(-\nabla_{N} \ln \phi+\delta\right)$ & \\
$V_{A}^{t}=\epsilon_{A}-\nabla_{A} \ln \phi=W_{A}-\nabla_{A} \ln (a \phi)$ \\
\hline \hline
\end{tabular}

\footnotetext{
${ }^{7}$ The physical meaning of this lapse function is discussed in [11]. In general, the lapse function, together with its counterpart, the shift vector, may be described as the nondynamical variable that tells one how to move forward in time (cf. e.g. $[12,1]$ ).
}

Hence, in terms of the geodesic null pair, $l=a^{-1} l^{\prime}, \underline{l}=a \underline{l}^{\prime}$ one obtains the results in Table II, where use is made of $D_{l} a=D_{a^{-1} l^{\prime}} a=-2 \Omega^{\prime} \Rightarrow D_{l^{\prime}} a=-2 a \Omega^{\prime}$ (derived using relations from [8], p. 264). In terms of the $t$-null pair, $l^{t}$ $=\phi l^{\prime}, \underline{l}^{t}=\phi^{-1} \underline{l}^{\prime}$, one gets the results in Table III.

\section{APPENDIX D: DECAY LAW FOR RICCI COEFFICIENTS}

This appendix gives the decays laws for the Ricci rotation coefficients using results from [8] and [10]. We will need the following: for any 2-symmetric covariant tensor, $H_{A B}$, on a topologically $S^{2}$ surface (p. 40 of [8]):

$$
H_{A B}=\hat{H}_{A B}+\frac{1}{2} \gamma_{A B} \operatorname{tr} H
$$

where the hat means the traceless part.

In the optical-maximal foliation using the geodesic null pair $(l, \underline{l})$ in a coordinate basis, the Ricci rotation coefficients (cf. Appendix B) near null infinity are (where $\epsilon>0$ ) seen in Table IV.

Note that the order of $\Omega$ can be found by using $a \propto 1$ $+\Psi^{\prime} / r$ and the fact that $\Psi^{\prime}$ is independent of $u$.

Using the standard null pair $\left(l^{\prime}, l^{\prime}\right)$ one has the results in Table V.

$$
\Rightarrow Z_{A}=E_{A}+\nabla_{A} \Psi^{\prime} \text { and } Z_{A}^{(2)}=E_{A}^{(2)}+\emptyset_{A} \Psi^{(2)}
$$

Using the $t$-null pair $\left(l^{t}, \underline{l}^{t}\right)$ one has the results in Table VI.

\section{APPENDIX E: DECAY AT NULL INFINITY GIVES DECAY AS FUNCTION OF $r$ ALONE}

A given geometric quantity, say $Q$, defined on space-time might decay like at null infinity (on a fixed $C_{u}$ as $t \rightarrow \infty$ ):

$$
Q \sim Q^{(0)}(u, \omega)+\frac{Q^{(1)}(u, \omega)}{r}+\frac{Q^{(2)}(u, \omega)}{r^{2}}+\cdots
$$

Here $\omega \in S^{2}$, and $u, t$ are as defined in the text. Assume all $Q^{(n)}$ finite $\forall u$.

Note here that the areal radius of $S_{t, u}$ can be written $r(t, u)$ and $Q$ can be written $Q(t, u)$.

To see that it is the same law on a fixed $\Sigma_{t}$ observe that for large enough $u$ (which also means large $r$ )

$$
r\left|Q^{(0)}(u, \omega)-Q(u, \omega)\right| \sim Q^{(1)}(u, \omega),
$$

where I use the fact that each point of $\Sigma_{t}$ is on one of the $C_{u}$ cones and that there must be a unique limit at spatial infinity. In words, on $\Sigma_{t}$, for large enough $r, Q$ is proportional to $1 / r$ to leading order with proportionality $Q^{(1)}(u, \omega)$. This is true 
TABLE IV. Geodesic null pair $(l, \underline{l})$.

\begin{tabular}{ll}
\hline \hline$\hat{H}_{A B}=\Sigma_{A B}+O\left(r^{-\epsilon}\right) ;$ & $\underline{\hat{H}}_{A B}=r \Xi_{A B}+O\left(r^{1-\epsilon}\right) ;$ \\
$\operatorname{tr} H=\frac{2}{r}+\frac{H}{r^{2}}+O\left(r^{-(2+\epsilon)}\right)$ & $\operatorname{tr} \underline{H}=-\frac{2}{r}+O\left(r^{-(1+\epsilon)}\right)$ \\
$Z Z_{A}=W_{A}=\frac{Z_{A}}{r}+\frac{z_{A}^{(2)}}{r^{2}}$ & $\underline{Z Z_{A}}=-\frac{Z_{A}}{r}+O\left(r^{-(1+\epsilon)}\right)$ \\
$+O\left(r^{-(2+\epsilon)}\right)$ & $\underline{Y_{A}}=-\frac{2 \downarrow_{A} \Psi^{\prime}}{r}+O\left(r^{-(1+\epsilon)}\right)$ \\
$Y_{A}=0$ & $\underline{\Omega}=O\left(r^{-2}\right)$ \\
$\Omega=0$ & \\
$V_{A} \equiv W_{A}=\frac{Z_{A}}{r}+\frac{Z_{A}^{(2)}}{r^{2}}+O\left(r^{-(2+\epsilon)}\right)$ & \\
\hline \hline
\end{tabular}

for every such quantity $Q$, and it is not necessary that the decay law at null infinity have integer powers of $1 / r$. If one wants to add or multiply many such quantities (as one does in the integrals in question in this paper), it is obvious that the resultant of such addition and multiplication will also obey these rules.

An intuitive, simplified, way of thinking about this is to recall that the choice of $t=0$ in the text was arbitrary. One can pick a starting $t$ as large as one wishes, and, in this way, get a large enough $t$ on the $S^{2}$ surface on the maximal slice, $\Sigma_{t}$, as one would like and thus everywhere out to null infinity.

The above arguments show that the decay laws for $t$ $\rightarrow \infty$ apply also in general for $r \rightarrow \infty$ with $t=$ const.

One can go further and note that for nonsingular $Q^{(1)}$, one can write a decay law that is a function of $r$ only. For example, in the above case, let $C \equiv\{\max$ over $u$ and $\omega$ of $\left.Q^{(1)}(u, \omega)\right\}$ and get
TABLE V. Standard null pair $\left(l^{\prime}, \underline{l}^{\prime}\right)$.

$$
\begin{array}{rlrl}
\hat{H}_{A B}^{\prime}=\Sigma_{A B}+O\left(r^{-\epsilon}\right) & \underline{H}_{A B}=r \Xi_{A B}+O\left(r^{1-\epsilon}\right) ; \\
\operatorname{tr} H^{\prime}=\frac{2}{r}+\frac{H+2 \Psi^{\prime}}{r^{2}} & & \operatorname{tr} \underline{H}=-\frac{2}{r}+O\left(r^{-(1+\epsilon)}\right) \\
Z Z_{A}^{\prime}=\frac{Z_{A}}{r}+\frac{Z_{A}^{(2)}}{r^{2}}+O\left(r^{-(2+\epsilon)}\right) & & \underline{Z Z_{A}^{\prime}}=-\frac{Z_{A}}{r}+O\left(r^{-(1+\epsilon)}\right) \\
Y_{A}^{\prime}=0 & & \underline{Y}_{A}^{\prime}=-\frac{2 \bigotimes_{A} \Psi^{\prime}}{r^{2}}+O\left(r^{-(1+\epsilon)}\right) \\
\Omega^{\prime}=O\left(r^{-2}\right) & \underline{\Omega^{\prime}}=O\left(r^{-2}\right) \\
V_{A}^{\prime} \equiv \epsilon_{A}=\frac{E_{A}}{r}+\frac{E_{A}^{(2)}}{r^{2}}+O\left(r^{-(2+\epsilon)}\right) &
\end{array}
$$

$$
Q(u, \omega) \sim Q^{0}+\frac{C}{r}
$$

where $C$ is a constant.

\section{APPENDIX F: PULLED BACK QUANTITIES}

In order for all of the work done here to take place on actual spheres of unit radius at null infinity, in terms of pulled back quantities, I will always use the appropriate diffeomorphism, divide by the appropriate power of the lumi-

\begin{tabular}{|c|c|}
\hline$\hat{H}_{A B}^{t}=\Sigma_{A B}+O\left(r^{-\epsilon}\right)$ & $\underline{\hat{H}}_{A B}^{t}=r \Xi_{A B}++O\left(r^{1-\epsilon}\right)$ \\
\hline $\operatorname{tr} H^{t}=\frac{2}{r}=\frac{H+2\left(\Psi+\Psi^{\prime}\right)}{r^{2}}+O\left(r^{-(2+\epsilon)}\right)$ & $\operatorname{tr} \underline{H}=-\frac{2}{r}+O\left(r^{-(1+\epsilon)}\right)$ \\
\hline$Z Z_{A}^{t}=\frac{Z_{A}}{r}+\frac{Z_{A}^{(2)}}{r^{2}}+O\left(r^{-(2+\epsilon)}\right)$ & $\underline{Z Z_{A}^{t}}=-\frac{Z_{A}}{r}+O\left(r^{-(1+\epsilon)}\right)$ \\
\hline$Y_{A}^{t}=0$ & $\underline{Y}_{A}^{t}=-\frac{2 \bigotimes_{A} \Psi^{\prime}}{r}+O\left(r^{-(1+\epsilon)}\right)$ \\
\hline$\Omega^{t}=O\left(r^{-2}\right)$ & $\underline{\Omega}^{t}=O\left(r^{-2}\right)$ \\
\hline$V_{A}^{t}=\frac{Z_{A}}{r}+\frac{Z_{A}^{(2)}}{r^{2}}+\frac{\bigotimes_{A} f}{r^{1+\epsilon}}$ & where $f$ is some particular function on $S^{2}$ \\
\hline
\end{tabular}
nosity (areal) radius defined by $r=\sqrt{\text { surface area/4 } \pi}$ and take the limit as $s \rightarrow \infty$ in the affine foliation. For example, the metric $\gamma$ on the topological sphere $S_{t, u}$ will have a diffeomorphism $\phi_{u, t} ; S^{2} \rightarrow S_{t, u}$; it takes one from an actual $S^{2}$ surface to the topological sphere $S_{t, u}$. One can use the pull back and show $\lim _{s \rightarrow \infty} \phi_{u, t}^{*} \gamma=\gamma^{0}$, the standard metric on the unit sphere. More details on this can be found in $[8,10,4,11]$.

TABLE VI. t-null pair $\left(l^{t}, \underline{l}^{t}\right)$. 
[1] Robert M. Wald, General Relativity (University of Chicago Press, Chicago, 1984).

[2] S. Deser, R. Arnowitt, and C. W. Misner, in Gravitation: An Introduction to Current Research, edited by L. Witten (Wiley, New York, 1962).

[3] S. Deser, R. Arnowitt, and C. W. Misner, Phys. Rev. 117, 1959 (1960); 118, 1100 (1960); 122, 997 (1961).

[4] A. Rizzi, Phys. Rev. Lett. 81, 1150 (1998).

[5] James W. York, Jr., in Essays in General Relativity, edited by Frank J. Tipler (Academic, New York, 1980), Chap. 4, pp. 39-57.

[6] General Relativity and Gravitation: One Hundred Years After the Birth of Albert Einstein, edited by A. Held (Plenum, New York, 1980), Vol. 2.
[7] Niall Ó Murchadha and James W. York, Jr., Phys. Rev. D 10, 428 (1974).

[8] D. Christodoulou and S. Klainerman, The Global Nonlinear Stability of the Minkowski Space (Princeton University Press, Princeton, NJ, 1993).

[9] Anthony Rizzi, in Proceedings of the Eighth Marcel Grossmann Meeting on General Relativity, edited by Tsvi Piran (World Scientific, Singapore, 1999), pp. 297-299.

[10] D. Christodoulou, Phys. Rev. Lett. 67, 1486 (1991).

[11] A. Rizzi, Ph.D. thesis, Princeton University, 1997.

[12] C. W. Misner, K. S. Thorne, and J. A. Wheeler, Gravitation (Freeman, San Francisco, 1973). 